\title{
Diretrizes para o Implante de Marcapasso Cardíaco Permanente
}

\author{
José Carlos S. Andrade, Vicente Ávila Neto, Domingo M. Braile, Paulo R. S. Brofman, Alvaro R. B. Costa, \\ Roberto Costa, Silas S. Galvão F, Paulo R. A. Gauch, Fernando A. Lucchese, Martino Martinelli F', \\ Paulo T. J. Medeiros, José C. P. Mateos, João Pimenta, Roberto T. Takeda
}

São Paulo, SP

\section{Introdução}

A estimulação cardíaca artificial nasceu no final dos anos 50, com o objetivo primordial de eliminar os sintomas e reduzir a mortalidade dos pacientes com bloqueios atrioventriculares (BAV) avançados. Essa finalidade foi conseguida já nas primeiras gerações de marcapassos, que inicialmente eram assincrônicos (VOO) e depois sincrônicos ou de demanda(VVI).

A partir daí, enorme avanço tecnológico ocorreu na fabricação dos geradores, com a utilização de microcircuitos integrados (alta capacidade de programabilidade e baixo consumo), baterias de lítio (grande durabilidade), fechamento hermético (aumento de segurança), multiprogramabilidade (diversidade de recursos), telemetria bidirecional (segurança e confiabilidade) sensores biológicos (possibilidade de alteração de frequiência). A evolução também foi observada na tecnologia dos cabos-eletrodos, desenvolvidos com menor calibre, maior flexibilidade, maior resistência, melhor histo-compatibilidade e fácil implantação por via venosa, tanto em câmaras atriais como ventriculares.

A associação do desenvolvimento da tecnologia de fabricação e de implante do marcapasso ao maior conhecimento eletrofisiopatológico dos distúrbios de condução cardíaca permitiu um progresso consistente na estimulação cardíaca artificial, extremamente diversificada no seu modo de funcionamento, com alta confiabilidade, segurança e cada vez mais fisiológica.

Ampliado o campo de ação dos marcapassos e diversificadas as suas indicações e modos de operação, as Sociedades que congregam os especialistas da área passaram a revisar periodicamente, as recomendações para a sua utilização, dadas as constantes inovações. Em nível nacional, o Departamento de Estimulação Cardíaca Artificial (Deca), da Sociedade Brasileira de Cirurgia Cardiovascular (SBCCV) emitiu em 1987 as "Diretrizes para Implante de Marcapasso Cardíaco Permanente". Em 1990, em conjunto com o Depar-

Consenso do Departamento de Estimulação Cardíaca Artificial da Sociedade Brasileira de Cirurgia Cardiovascular, 1999

Correspondência: Paulo R. A. Gauch - DECA - Rua Beira Rio, $45-7^{\circ}-\operatorname{conj} 73$ - 04548-050 - São Paulo, SP tamento de Arritmia e Eletrofisiologia Clínica (Daec), tal trabalho, revisto e atualizado, foi publicado sob o título: "Indicações para Implante de Marcapasso Cardíaco Permanente". Cinco anos após, outra publicação do Deca modernizou as diretrizes para utilização da estimulação cardíaca artificial: "Consenso para o Implante de Marcapasso Cardíaco Permanente e Cardioversor-Desfibrilador Implantável - 1995".

A presente atualização foi motivada pelo extraordinário e rápido desenvolvimento da estimulação cardíaca artificial no tratamento das bradi e taquiarritmias, como também na terapêutica das miocardiopatias hipertróficas e dilatadas, correção dos distúrbios hemodinâmicos, ressincronização de câmaras cardíacas, prevenção de taquiarritmias e monitoração cardiovascular diagnóstica.

\section{Indicações}

Seguindo o mesma orientação dos trabalhos anteriores, as várias situações clínicas em que se discute o implante de marcapasso cardíaco permanente foram classificados em: classe I - situações em que existe concordância geral quanto à indicação do implante de marcapasso; classe II - situações em que freqüentemente há indicação de estimulação artificial, mas nas quais não existe concordância geral quanto à sua necessidade absoluta. Nessas condições, a ampla avaliação, o senso clínico, a experiência e a individualização de cada decisão poderão incluir ou excluir certo número de casos; classe III - situações em que há concordância geral de que o implante de marcapasso não é necessário.

\section{Bloqueio atrioventricular do $3^{o}$ grau}

Classe I - 1) Bloqueio atrioventricular total (BAVT) permanente ou intermitente, irreversível, de qualquer etiologia ou localização, com sintomas definidos de baixo débito cerebral e/ou insuficiência cardíaca, conseqüentes à bradicardia; 2) BAVT assintomático, conseqüente a infarto agudo do miocárdio, persistente por mais de 15 dias; 3 ) BAVT assintomático, conseqüente a cirurgia cardíaca, persistente por mais de 15 dias, com QRS largo; 4) BAVT assintomático, conseqüente a cirurgia cardíaca, persistente por mais de 15 dias, com QRS estreito e ritmo de escape infranodal; 5) BAVT assintomático, irreversível, de localização intra ou infra-His, ou com ritmo de escape infra-His; 6) 
BAVT assintomático, irreversível, mesmo com QRS estreito, com arritmias ventriculares que necessitem de antiarrítmicos depressores do ritmo de escape; 7) BAVT adquirido, irreversível, assintomático, com freqüência cardíaca média inferior a 40bpm na vigília e sem aceleração adequada ao exercício; 8) BAVT irreversível, assintomático, com períodos documentados de assistolia acima de 3 segundos na vigília; 9) BAVT irreversível, assintomático e com cardiomegalia progressiva; 10) BAVT congênito, assintomático, com ritmo de escape de QRS largo (superior a 120ms) ou com frequiência cardíaca inadequada para a idade; 11) BAVT adquirido, assintomático, de etiologia chagásica ou esclerodegenerativa; 12) BAVT irreversível, permanente ou intermitente, conseqüente à ablação da junção atrioventricular.

Classe II - 1) BAVT conseqüente a cirurgia cardíaca, assintomático, persistente após 15 dias, com QRS estreito ou ritmo de escape nodal e boa resposta cronotrópica; 2) BAVT conseqüente a cirurgia cardíaca ou infarto agudo do miocárdio, sem perspectiva de reversão; 3) BAVT congênito, com QRS estreito, aceleração adequada ao exercício e sem cardiomegalia, mas com arritmia ou QT longo.

Classe III - 1) BAVT congênito, assintomático, com QRS estreito, com aceleração adequada ao exercício e sem cardiomegalia, arritmia ou QT longo; 2) BAVT transitório por ação medicamentosa ou química, processo inflamatório agudo, cirurgia cardíaca ou outra causa reversível.

\section{Bloqueio atrioventricular do $2^{\circ}$ grau}

Classe I-1) Bloqueio atrioventricular (BAV) $2^{\circ}$ grau, permanente ou intermitente, irreversível ou causado por drogas necessárias e insubstituíveis, independentemente do tipo e localização, com sintomas definidos de baixo fluxo cerebral e/ ou insuficiência cardíaca, conseqüentes à bradicardia; 2) BAV $2^{\circ}$ grau, tipo II, com QRS largo ou infra-His, assintomático, permanente ou intermitente e irreversível; 3) Flutter ou fibrilação atrial, com períodos de resposta ventricular baixa, em pacientes com sintomas definidos de baixo fluxocerebral e/ou insuficiência cardíaca conseqüentes à bradicardia.

Classe II - 1) BAV $2^{\circ}$ grau avançado, adquirido, assintomático, permanente ou intermitente e irreversível; 2) BAV $2^{\circ}$ grau, tipo II, com $Q R S$ estreito, assintomático, permanente ou intermitente e irreversível; 3) BAV $2^{\circ}$ grau 2:1, assintomático, permanente ou intermitente e irreversível; 4) BAV $2^{\circ}$ grau 2:1, com QRS estreito, assintomático, persistente após 15 dias de cirurgia cardíaca ou infarto agudo do miocárdio;5) BAV $2^{\circ}$ grau irreversível, assintomático, associado a arritmias ventriculares que necessitam de tratamento com fármacos insubstituíveis, depressores da condução atrioventricular; 6) Flutter ou fibrilação atrial, assintomático, com freqüência ventricular média inferior a 40 bpm na vigília, irreversível ou por uso de fármaco necessário e insubstituível.

Classe III -1) BAV $2^{\circ}$ grau tipo I, assintomático, com aumento da frequiência cardíaca e melhora da condução atrioventricular com exercício e/ou atropina intravenosa.

\section{Bloqueio atrioventricular do $1^{\circ}$ grau}

Classe I-Nenhuma.

Classe II-1) BAV $1^{\circ}$ grau, irreversível, com síncopes, pré-síncopes ou tonturas e cujo estudo eletrofisiológico denota localização intra ou infra-His e agravamento do mesmo por estimulação atrial ou teste farmacológico.

Classe III-1) BAV $1^{\circ}$ grau assintomático.

\section{Bloqueios intraventriculares}

Classe I - 1) Bloqueio de ramo alternante com síncopes, pré-síncopes ou tonturas recorrentes.

Classe II-1) Bloqueios intraventriculares com intervalo $\mathrm{HV}$ igual ou superior a $70 \mathrm{~ms}$ ou com bloqueio intra ou infra-His induzido por estimulação atrial e/ou teste farmacológico, em pacientes com síncopes, pré-síncopes ou tonturas recorrentes sem causa determinada; 2 ) bloqueios intraventriculares em pacientes assintomáticos com intervalo HV igual ou superior a $100 \mathrm{~ms}$; 3 ) bloqueio bifascicular, associado ou não a $\mathrm{BAV}$ de $1^{\circ}$ grau, com episódios sincopais sem prova da existência de BAVT, paroxístico, tendo sido afastadas outras causas para os sintomas; 4) bloqueio de ramo alternante, assintomático.

Classe III - 1) Bloqueios uni ou bifasciculares, assintomáticos, de qualquer etiologia.

\section{Doença do nó sinusal}

Classe I - 1) Disfunção do nó sinusal, espontânea ou induzida por fármacos necessários e insubstituíveis, com síncopes, pré-síncopes ou tonturas e/ou insuficiência cardíaca relacionadas à bradicardia; 2) síndrome bradi-taqui.

Classe II-1) Disfunção do nó sinusal, irreversível ou induzida por fármacos necessários e insubstituíveis, com sintomas de baixo fluxo cerebral não claramente relacionados com a bradicardia, tendo sido afastadas outras causas para os sintomas; 2) disfunção do nó sinusal, com intolerância aos esforços claramente relacionada à incompetência cronotrópica; 3 ) bradiarritmia sinusal que desencadeia ou agrava insuficiência cardíaca congestiva, angina do peito ou taquiarritmias; 4) bradi-taquiarritmia assintomática.

Classe III - 1) Disfunção do nó sinusal em pacientes assintomáticos; 2) disfunção do nó sinusal com sintomas comprovadamente independentes da bradicardia.

\section{Síndromes neuro-mediadas}

Classe I - 1) Pacientes com síncopes espontâneas e repetitivas, associadas a ocorrências claramente produtoras de estimulação do seio carotídeo, nos quais manobras provocativas mínimas produzem assistolia superior a $3 \mathrm{~s}$, na ausência de medicação depressora da função sinusal ou da condução atrioventricular; 2) pacientes com síncopes espontâneas e repetitivas, nas quais a massagem do seio carotídeo provoca assistolia superior a três segundos, com reprodutibilidade dos sintomas, na ausência de medicação 
depressora da função sinusal ou da condução atrioventricular; 3) pacientes com síncope neurocardiogênica com importante componente cárdio-inibidor, documentado com teste de inclinação (tilt-test), claramente refratária ao tratamento farmacológico.

Classe II - 1) Pacientes com síncopes ou pré-síncopes espontâneas e repetitivas, nos quais a massagem do seio carotídeo provoca assistolias superior a $3 \mathrm{~s}$, sem reprodutibilidade da sintomatologia; 2) pacientes com síncopes neurocardiogênica com importante componente cárdio-inibidor, documentado no teste de inclinação (tilt-test), com resposta inadequada ou insuficiente ao tratamento farmacológico.

Classe III - 1) Pacientes nos quais a massagem do seio carotídeo provoca assistolia inferior a $3 \mathrm{~s}$, não se conseguindo atribuir origem cardiovascular à sintomatologia; 2) pacientes sintomáticos nos quais a massagem do seio carotídeo identifica apenas resposta vasodepressora; 3) pacientes assintomáticos nos quais a massagem do seio carotídeo provoca assistolia, com ou sem aparecimento de sintomatologia; 4) pacientes com síncope neurocardiogênica com componente cárdio-inibidor, documentado com teste de inclinação (tilt-test), com resposta adequada ao tratamento farmacológico; 5) pacientes com síncope neurocardiogênica do tipo vasodepressora; 6) pacientes com síncopes situacionais, provocadas por micção, tosse, deglutição, mergulho, etc.

\section{Síncopes recorrentes de origem desconhecida}

Classe I - Nenhuma.

Classe II - 1) Síncopes em pacientes nos quais se demonstra intervalo $\mathrm{HV}$ igual ou superior a $70 \mathrm{~ms}$ ou se induz BAV paroxístico de $2^{\circ}$ ou $3^{\circ}$ graus, de localização intra ou infra-His, por estimulação atrial ou teste farmacológico.

Classe III - 1) Síncopes em pacientes nos quais não se consegue atribuir origem cardíaca aos sintomas.

\section{Indicações especiais}

\section{Classe I - Nenhuma.}

Classe II - 1) Pacientes portadores de cardiomiopatia hipertrófica obstrutiva, sintomáticos, refratários ao tratamento farmacológico; 2) pacientes portadores de síndrome do QT longo, com tratamento farmacológico contra-indicado ou insuficiência para o controle das arritmias; 3) pacientes com cardiomiopatia dilatada, insuficiência cardíaca congestiva (classe funcional III/IV NYHA), refratários ao tratamento farmacológico, com BRE e/ou PR prolongado; 4) $\mathrm{BAV} 1^{\circ}$ grau, permanente ou intermitente, irreversível ou induzido por fármacos necessários e insubstituíveis, com sintomatologia limitante, comprovadamente relacionada à contração atrioventricular simultânea.

Classe III-1) Pacientes com cardiomiopatiahipertrófica obstrutiva, assintomáticos ou controlados por terapêutica medicamentosa; 2) pacientes com cardiomiopatia hipertrófica não obstrutiva; 3 ) pacientes assintomáticos, com cardiomiopatia dilatada; 4) pacientes sintomáticos, com car- diomiopatia dilatada, controlados por terapêutica medicamentosa; 5) pacientes com cardiomiopatia dilatada isquêmica.

\section{Modos de estimulação}

A escolha do modo de estimulação deve envolver a obediência aos princípios fisiológicos, mas também respeitar a individualidade de cada paciente e os recursos pessoais e materiais disponíveis no serviço.

Idealmente deve-se procurar manter o sincronismo atrioventricular (AV), obtendo-se a variação da frequiência de estimulação ventricular com a sincronização dessa estimulação à variação da frequiência de $\mathrm{P}$ num nó sinusal que apresente cronotropismo conservado ou com a utilização de um biossensor que, captando as necessidades metabólicas, modifique artificialmente a freqüência de estimulação já na câmara atrial. Os seguintes princípios são aconselháveis: - a estimulação ventricular deverá ser utilizada sempre que houver presença ou risco de $\mathrm{BAV}$; - o sincronismo atrioventricular deverá ser preservado sempre que possível; - a estimulação atrial terá indicação na ausência de cronotropismo positivo no nó sinusal; - a resposta de freqüência deverá ser perseguida pela utilização da variação de freqüência ditada por um nó sinusal normal ou pela resposta de um biossensor; - o biossensor preferencialmente deverá apresentar um sistema de alça fechada.

Quanto às câmaras e sítios a serem estimulados a forma de estimulação, deve propiciar a otimização hemodinâmica, objetivando a estabilidade elétrica e a melhora do débito cardíaco.

\section{AAI - Estimulação atrial monocâmara}

Indicação - Doença do nó sinusal sem bloqueio atrioventricular e sem incompetência cronotrópica.

Contra-indicações - Bloqueio atrioventricular; taquiarritmia atrial persistente; síndrome do seio carotídeo hipersensível e/ou síncope neurocardiogênica; doença do nó sinusal com necessidade de resposta cronotrópica.

Vantagens - Cabo-eletrodo único; marcapasso monocâmara padrão; manutenção do sincronismo atrioventricular.

Desvantagem - Ausência de proteção no surgimento de bloqueio atrioventricular ou incompetência cronotrópica.

\section{AAIR - Estimulação atrial monocâmara com resposta de freqüiência}

Indicação - Doença do nó sinusal sem bloqueio atrioventricular, com incompetência cronotrópica.

Contra-indicações - Bloqueio atrioventricular; taquiarritmia atrial persistente; síndrome do seio carotídeo hipersensível e/ou síncope neurocardiogênica.

Vantagens - Cabo-eletrodo único; manutenção do sincronismo atrioventricular; resposta de freqüência

Desvantagens - Ausência de proteção no surgimento de bloqueio atrioventricular; síndrome do marcapasso em pacientes com prolongamento da condução atrioventri- 
cular ao exercício; possibilidade de bloqueio atrioventricular com elevação da resposta de freqüência; cabo-eletrodo especial em alguns tipos de sensores.

\section{VVI - Estimulação ventricular monocâmara}

Indicações - Fibrilação/flutter e outras taquiarritmias atriais persistentes com bloqueio atrioventricular; bloqueio atrioventricular em crianças.

Contra-indicações - Síndrome do marcapasso; doença do nó sinusal; síndrome do seio carotídeo hipersensível; síncope neurocardiogênica.

Vantagens - Cabo-eletrodo único; marcapasso monocâmara padrão

Desvantagens - Impossibilidade de sincronismo atrioventricular normal com predisposição à síndrome do marcapasso; fibrilação atrial e tromboembolismo; ausência de resposta de frequiência.

\section{VVIR - Estimulação ventricular monocâmara com res- posta de freqüência}

Indicações - Fibrilação/flutter e outras taquiarritmias atriais persistentes, com bloqueio atrioventricular adquirido ou provocado; bloqueio atrioventricular em crianças e/ou pacientes com limitações ao uso do DDD.

Contra-indicações - Síndrome do marcapasso; doença do nó sinusal; síndrome do seio carotídeo hipersensível; síncope neurocardiogênica.

Vantagens - Cabo-eletrodoúnico; resposta de freqüência.

Desvantagens - Impossibilidade de sincronismo atrioventricular normal, com predisposição à síndrome do marcapasso; fibrilação atrial e tromboembolismo.

\section{DDI - Estimulação de dupla-câmara sem deflagração ventricular pelo átrio}

Indicações - Síndrome do seio carotídeo hipersensível; síncope neurocardiogênica; doença do nó sinusal com paroxismo de taquiarritmia atrial.

Contra-indicações - Bloqueio atrioventricular isolado; taquiarritmia atrial persistente.

Vantagens - Manutenção do sincronismo atrioventricular; proteção do surgimento de bloqueio atrioventricular; impossibilidade de taquicardias mediadas; impossibilidade de deflagração ventricular por taquicardias atriais.

Desvantagens - Ausência de resposta de freqüência; necessidade de eletrodos atriais; possibilidade de síndrome do marcapasso

\section{DDIR - Estimulação de dupla-câmara sem deflagração ventricular pelo átrio com resposta de freqüiência}

Indicação - Doença do nó sinusal com incompetência cronotrópica ou com paroxismo de taquiarritmia atrial.

Contra-indicações - Bloqueios atrioventriculares isolados; taquiarritmias atriais persistentes.

Vantagens - Manutenção do sincronismo atrioventri- cular; resposta de freqüência; ausência de taquicardias mediadas; ausência de deflagração ventricular de taquiarritmias atriais.

Desvantagens - Necessidade de eletrodos atriais; possibilidade de síndrome do marcapasso.

\section{DDD - Estimulação dupla-câmara com deflagração ventricular pelo átrio}

Indicações - Bradiarritmias permanentes ou intermitentes, com cronotropismo preservado; bloqueio atrioventricular com função sinusal normal; situações especiais

Contra-indicação - Taquiarritmias atriais persistentes

Vantagens - Manutenção do sincronismo atrioventricular; preservação da resposta natural de frequiência.

Desvantagens - Necessidade de eletrodos atriais; possibilidade de taquicardias mediadas; possibilidade de comportamento não fisiológico no limite de freqüência máxima do marcapasso; possibilidade de deflagração ventricular de taquiarritmias atriais.

\section{DDDR - Estimulação dupla-câmara com deflagração ventricular pelo átrio com resposta de freqüiência}

Indicação - Bradiarritmias permanentes ou intermitentes com ausência de resposta de freqüência;

Contra-indicação - Taquiarritmias atriais persistentes.

Vantagens - Manutenção do sincronismo atrioventricular; Deflagração ventricular peloátrio; resposta de freqüência.

Desvantagens - Necessidade de eletrodos atriais; possibilidade de taquicardias mediadas; possibilidade de deflagração ventricular de taquiarritmias atriais.

\section{VDD - Estimulação ventricular deflagrada pelo átrio sem possibilidade de estimulação atrial}

Indicação - Bloqueio atrioventricular com função sinusal normal.

Contra-indicações - Doença do nó sinusal; taquiarritmias atriais; síndrome do seio carotídeo hipersensível; síncope neurocardiogênica.

Vantagens - Manutenção do sincronismo atrioventricular; resposta natural de freqüência; possibilidade da utilização de eletrodo único.

Desvantagens - Possibilidade de síndrome do marcapasso; impossibilidade de estimulação atrial.

\section{Modos especiais de estimulação}

\section{A - Estimulação atrial multi-sítio}

Indicação - Distúrbio da condução intra e/ou interatrial em portadores de instabilidade elétrica atrial e/ou comprometimento hemodinâmico conseqüente a retardo na ativação atrial esquerda.

Contra-indicações - Fibrilação atrial permanente e irreversível; paralisia atrial. 
Vantagens - Ressincronização atrial; redução do tempo de ativação atrial.

Desvantagem - Necessidade de implante de mais eletrodos.

\section{B - Estimulação ventricular multi-sítio}

Indicações - Cardiomiopatia dilatada com bloqueio de ramo esquerdo e insuficiência cardíaca (classe III/IV NYHA) de difícil controle medicamentoso. Fibrilação atrial permanente e irreversível com bloqueio atrioventricular espontâneo ou induzido por ablação em portador de cardiomiopatia dilatada com insuficiência cardíaca de difícil controle medicamentoso (classe III/IV NYHA).

Contra-indicação-Nenhuma.

Vantagens - Redução do tempo de ativação ventricular; melhora da função ventricular; possibilidade de redução da regurgitação mitral.

Desvantagens - Necessidade de mais eletrodos; dificuldade de acesso ao ventrículo esquerdo.

\section{C-Cardiomioestimulação}

Indicação - Pacientes com insuficiência cardíaca congestiva, classe funcional III ou IV NYHA, com fração de ejeção menor ou igual a 35\%, diâmetro diastólico final de ventrículo esquerdo $<75 \mathrm{~mm}$ (ecocardiograma), sem hipertensão arterial pulmonar.

Contra-indicações - Falência de múltiplos órgãos; doenças musculares degenerativas.

Vantagens-Redução da classe funcional de insuficiência cardíaca congestiva; melhora da fração de ejeção pela estimulação sincrônica, como músculo esquelético translocado.

Desvantagem - Porte cirúrgico.

\section{D - Monitoração endocavitária ou epimiocárdica trans- telemétrica do coração transplantado}

Procedimento diagnóstico para acompanhamento de processos de rejeição, infecciosos ou inflamatórios, em transplantados de coração e em miocardiopatas, alvissareiro mas ainda em fase de investigação.

\section{Indicações para a troca de gerador}

1) Desgaste de bateria com sinais de fim de vida; 2 ) ausência ou queda do pulso de saída; 3 ) falha no circuito de sensibilidade; 4) contaminação ou infecção; 5) recall; 6) oportunidade cirúrgica, tendo sido ultrapassado o tempo de garantia do gerador; 7) defeito no conector; 8) presença de síndrome do marcapasso; 9) estimulação muscular não corrigível por programação.

\section{Indicações para a troca de eletrodo}

1) Ruptura de isolamento; 2) fratura do condutor; 3 ) recall;4) contaminação ou infecção; 5) alteração significativa da impedância; 6) indicação clínica de substituição do modo de estimulação (uni para bipolar) em cabo unipolar; 7) infiltração; 8) repetidos deslocamentos ou elevações de limiares.

\section{Indicações para a reoperação com possibili- dade de reposição ou troca de eletrodo}

1) Deslocamento; 2) aumento acentuado do limiar crônico de estimulação ou perda de comando; 3 ) diminuição acentuada ou perda da sensibilidade; 4) risco de escara ou protusão cutânea; 5) estimulação frênica ou diafragmática; 6) perfuração ventricular.

\section{Indicações especiais para a troca de gerador com cabo-eletrodo}

1) Pacientes com sintomas ou sinais decorrentes de modo de estimulação inadequado cuja resolução é impossível por reprogramação; 2) portadores sintomáticos de marcapassos não responsivos, com necessidade documentada de resposta de freqüência; 3) portadores sintomáticos de marcapassos responsivos, mas com resposta inadequada do sensor; 4) portadores de marcapassos convencionais cuja evolução clínica mostra necessidade de estimulação multi-sítio.

\section{Artigos consultados}

\footnotetext{
Alpert MA, Curtis JJ, et al. Comparative survival following permanent ventricular and dual-chamber pacing for patients with chronic symptomatic sinus node dysfunction with and without congestive heart failure. Am Heart J 1987; 113. Ausubel K, Furman S. The pacemaker syndrome. Ann Intern Med 1985: 420-9. Barold SS, Mugica J. Estimular ou sentir oátrio, salvo contra-indicações: ...Regra de ouro da estimulação cardíaca dos anos 90. Rev Bras Marcapasso e Arritmia 1994; 7: 107-18.

Berder V, Daubert C, Mabo P, De Place C, Gras D, Leclercq C. La resynchronisation atriale permanente dans les blocs inter auriculares de haut degré: aspects électrophysiologiques et hémodynamiques. Stimucoeur 1993; 21: 157-62. Cazeau S, Ritter P. Les indications de la stimulation cardiaque em 1991: analyse bibliographique des recommandation de l' AHA. Stimucoeur 1992; 20: $72-7$.
}

\footnotetext{
Clarke M, Sutton R, Ward D, Camn AJ. et al. Recommendations for pacemaker prescription for symptomatic bradycardia. Br Heart J 1991; 66: 185-91. Consenso para o implante de marcapasso cardíaco permanente e desfibriladorcardioversor-implantável - 1995 (Deca/SBCCV). Reblampa 1995; 8: 4-11. Daubert JC, Leclercq C, Pavin D, Mabo P. Pacing therapy in congestive heart failure. In: Barold SS, Mugica J. Recent advances in cardiac pacing: goals for the $21^{\text {st }}$ century. Armonk, NY: Futura Publishing, 1988: 51-80.

- Daubert C, Mabo P, Gras D, Leclercq C. Quelle place reste-t-il pour la stimulation monochambre ventriculaire? Stimucoeur 1993; 21: 89-97.

Daubert JC, Mabo P, Ritter P. Les principaux determinantes de la performance cardiaque en stimulation permanent. Stimucoeur 1994; 22: 73-86.

Diretrizes para implante de marcapasso cardíaco permanente do Departamento de
} 
Estimulação Cardíaca Artificial da Sociedade Brasileira de Cirurgia Cardiovascular. Rev. Bras. de Marcapasso e Arritmia 1988; 1: 23-6.

Dreifus LS, Fisch C, Griffin JC, Gillette PC, et al. Guidelines for implantation of cardiac pacemaker and antiarrhythmia devices. Circulation 1991; 84: 455-67.

- Fananapazir L, Epistein ND, Curiel RV, et al. Long term results of dual chamber (DDD) pacing in obstructive hypertrophic cardiomyopathy. Circulation 1994; 90: 2731-42.

- $\quad$ Fananapazir L, Mc Areavey D. Therapeutic options in patients with obstructive hypertrophic cardiomyopathy and severe drug-refractary symptoms. J Am Coll Cardiol 1998; 31: 259-64.

- Hochleitner M, Hortnagl H, et al. Usefulness of physiologic dual-chamber pacing in drug-resistant idiopathic dilated cardiomyopathy. Am J Cardiol 1990; 66: 198-202.

- Hochleintner M, Hornagl N, Hortnagl H, et al. Long-term efficacy in the treatment of end-stage idiopathic dilated cardiomyopathy. Am J Cardiol 1992; 70: 1320-5. Indicações para implante de marcapasso cardíaco permanente. Deca/Daec 1990. Rev Bras de Marcapasso e Arritmia 1990; 3.

- Mahaux V, Legrand V, Walefee A, et al. Acute hemodynamics of biventricular sti- mulation in congestive heart failure. In: Oto A. Europace 95; $7^{\text {th }}$ European Symposium on Cardiac Pacing. Istanbul. Turkey, June 4-7, 1995, Bologna, Italy: Monduzzi Editore, 1995: 359-62.

- Mahaux V, Demoulin JC, Pierard L, et al. Biventricular stimulation in congestive heart failure: initial clinical experience. In: Oto A. Europace 95, 7th European Symposium on Cardiac Pacing. Istanbul, Turkey, June 4-7, 1995, Bologna, Italy: Monduzzi Editore, 1995: 533-6.

- $\quad$ Rosenqvist M, Brandt J, Scüller H. Long-term pacing and sinus node disease: effects of stimulation mode on cardiovascular morbidity and mortality. Am Heart J 1988; 118: 16-21.

- Sulke N, Chambers J, et al. A randomized double-blind crossober comparison of four rate-responsive pacing modes. J Am Coll Cardiol 1991; 17: 696-706.

- Travill CM, Sutton R. Pacemaker syndrome: an iatrogenic condition. Br Heart J 1992; 68: 163-6.

- TripoldD, Fananapazir L. Five years results of dual chamber(DDD) pacing for severe drug-refractary symptons in obstructive hypertrophic cardiomyopathy. PACE 1997; 20(II): 1102. 